Original

\title{
Complicaciones en cirugía laparoscópica renal en la edad pediátrica: análisis de nuestra experiencia y revisión de la literatura
}

\author{
Alberto Pérez-Lanzac de Lorca*, Andrés Gómez Fraile**, Adolfo Aransay Bramtot**, \\ Daniel Cabezalí Brabancho**, Francisco López Vázquez**, Jesús Castiñeiras Fernández* \\ *Servicio de Urología. Hospital Universitario Virgen Macarena. Sevilla, España. **Sección de Urología \\ Pediátrica y Servicio de Cirugía Pediátrica. Hospital Materno-Infantil 12 de Octubre. Madrid, España.
}

\begin{abstract}
Resumen
Introducción: El desarrollo de la cirugía mínimamente invasiva en la edad pediátrica ha sido más lento que en la edad adulta ya que sus ventajas no son tan claras. El aprendizaje y las complicaciones presentadas durante este periodo, así como el tipo de material utilizado han sido los factores que han contribuido a este hecho.

Objetivo: Mostrar nuestra experiencia y las complicaciones presentadas en la cirugía laparoscópica de exéresis renal, añadiendo una revisión bibliográfica de las publicadas en esta edad hasta la fecha.

Material y Métodos: Estudio retrospectivo de nuestros casos clínicos en que practicamos cirugía renal laparoscópica extirpativa. Encontramos 56 casos desde Enero de 2003, en que se implantó, hasta Septiembre del 2008. Hubo 36 niños (64\%) y 20 niñas (36\%). La edad media de intervención fue de 3,6 años. Analizamos el diagnóstico principal, la situación que condujo a la intervención así como el tipo de cirugía efectuada, vía de abordaje, tiempo utilizado, tiempo de ingreso, complicaciones y tratamiento aplicado. Los resultados se analizaron mediante el programa estadístico SPSS ${ }^{\circledR}$ (SPSSCorp, Chicago, Illinois). La revisión bibliográfica se efectuó en las bases de datos MEDLINE, EMBASE y COCHRANE. Los trabajos seleccionados fueron revisados por dos investigadores. Series que contuvieran adultos fueron excluidas.

Resultados: Se han efectuado 42 nefrectomías (75\%), 13 heminefrectomías (23\%) y 1 quistectomía (2\%). En 5 casos (9\%) utilizamos la retroperitoneoscopia y en 51 (91\%) la vía transperitoneal. El tiempo medio quirúrgico fue de 118+/-0,75 min, para las nefrectomías, de 192+/-1,07 min., para las nefrectomías parciales, $111+/-0,64$ min para las nefroureterectomías y de 240 minutos para la quistectomía. La estancia media ha sido de 3,18 (2-6) días para las nefrectomías, 5,91 (3-11) días para las nefrectomías parciales, 3 días (2-4) para las nefroureterectomías y 6 días para la quistectomía. Hemos tenido 8 complicaciones (14\%): 2 reconversiones por sangrado y dificultad técnica y 6 postoperatorias (3 fueron menores, fiebre durante el postoperatorio y 3 mayores (2 Urinomas y un pseudoaneurisma, requiriendo 2 intervenciones y una colocación de catéter doble J). La evolución ha sido buena en todos los casos. En la revisión bibliográfica se seleccionaron 47 artículos con un nivel de evidencia IIIB (Oxford Centre for Evidence-based Medicine). El número de nefrectomías encontrado ha sido de 347 pacientes. Las causa más frecuente de reconversión fueron los problemas vasculares y las complicaciones variaron de ninguna hasta el $37 \%$.

Conclusiones: La cirugía laparoscópica ha demostrado ser una técnica segura y fiable en el tratamiento de la patología renal benigna de la edad pediátrica con buenos resultados. Actualmente sus indicaciones se están ampliando a procesos de reconstrucción, donde muestran unos resultados prometedores, y a procesos oncológicos seleccionados.
\end{abstract}

Palabras claves. Laparoscopia. Infancia. Complicaciones.

\section{Complications in laparoscopy renal surgery in pediatric age: Analysis of our experience and literature review}

\section{Abtract}

Introduction: The development of the minimally invasive surgery in pediatric age has been slower than in the adult age since their advantages are not so clear. The learning curve and the complications presented during this period, as well as the type of material used has been the factors that have contributed to this fact.

Objective: We collected our experience and the complications presented in renal laparoscopic exegesis surgery, adding a review of the published literature to date. Materials and Methods: Retrospective study of our clinical cases in that we practiced laparoscopic renal exegesis surgery. We collected 56 cases from January 2003, when it was implanted, to September 2008. We had 36 boys (64\%) and 20 girls (36\%). The mean age was 3.6 years. We analyzed principal diagnosis, the situation that conducted to the surgery and the type of surgery performed, time of surgery, hospital stay and complications with their solution. The results were analyzed by the statistical program SPSS $®$ (SPSSCorp, Chicago, Illinois). The literature review was practiced in MEDLINE, EMBASE and COCHRANE database. The selected works were reviewed by two investigators. Series containing adults were excluded.

Results: We perfomed 42 nephrectomies (75\%), 13 heminephrectomies (23\%) and 1 quistectomy (2\%). 5 cases (9\%) were done by retroperitoneoscopy and 51 cases (91\%) transperitoneally. The mean operative time was $118+/-0,75$ min, for nephrectomies; 192+/-1,07 min, for partial nephrectomies, $111+/-0,64$ for nephroureterectomies and $240 \mathrm{~min}$ for quistectomía. The mean hospital stay was 3.18 min (2-6) days for nephrectomies; 5.91 (3-11) days for partial nephrectomies, 3 days (2-4) for nephroureterectomies and 6 days for the quistectomy. We had 8 complications (14\%): 2 conversions for bledding and technical difficulty and 6 postoperative complications ( 3 were minor complications, postoperative fever and 3 mayor ( 2 Urinomas and one pseudoaneurism, requiring 2 interventions and one catheter double $\mathrm{J}$ placement). The evolution has been satisfactory in all cases. In the bibliographic review 47 articles with a level of evidence IIIB were selected (Oxford Centers for Evidence-based Medicine). The main reasons to conversion to open surgery were vascular problems and the complication rate change from anyone to $37 \%$.

Conclusions: Laparoscopic surgery has proved to be a secure and feasible technique in the treatment of benign renal pathology in pediatric age with satisfactory results. Actually the indications are expanding to reconstructive procedures, with promising results, and selected oncologic procedures.

Keywords: Laparoscopy. Childhood. Complications. 
T a implantación de la cirugía mínimamente invaLsiva en la edad pediátrica ha sido lenta pero progresiva. Las ventajas vistas en la edad adulta no son tan claras en los niños ${ }^{3,9}$ y cuestiones técnicas, como el aprendizaje, disposición de material adecuado o coste de los recursos están detrás de su pausado desarrollo. Desde que Clayman et al. ${ }^{1}$ publicarán la primera nefrectomía laparoscópica infantil en 1990 y después Jordan y Winslow ${ }^{2}$ en 1993 describieran la primera heminefrectomía laparoscópica en una duplicidad renal, se han publicado numerosas series de intervenciones laparoscópicas, que demuestran a esta técnica como una alternativa segura aunque no exenta de complicaciones. Se ha descrito una morbilidad no desdeñable en torno al $2,8 \%{ }^{3}$ y una curva de aprendizaje compleja con una tasa de reconversiones de hasta el $18 \%{ }^{4,5}$, según las series.

El objetivo del estudio es analizar nuestros casos, viendo diferentes aspectos de esta técnica, principalmente sus complicaciones, así como realizar una revisión bibliográfica de las distintas publicaciones de la cirugía renal laparoscópica extirpativa, en niños, analizando vía de abordaje y complicaciones referidas, así como su comparación con la cirugía abierta.

\section{MATERIAL Y MÉTODOS}

Estudio retrospectivo de los casos clínicos tratados por nuestra Sección de Urología Infantil mediante Laparoscopia. Hemos controlado y tratado mediante cirugía mínimamente invasiva 56 niños desde el 2003 hasta Septiembre del 2008 en los que se practicó cirugía renal extirpativa. De ellos, 36 (64\%) fueron niños y 20 niñas (36\%). Se recoge el diagnóstico principal, indicación quirúrgica, vía de abordaje, edad a la cirugía, tiempo de cirugía, estancia media hospitalaria, complicaciones y tratamiento aplicado para su resolución. Clínicamente todos los pacientes fueron evaluados previamente a la intervención mediante ECO y Renograma isotópico diurético (MAG-3) o gammagrafía renal (DMSA), dependiendo del tipo de patología que presentaban.

Técnicamente hemos utilizado dos vías de abordaje:

\section{Vía transperitoneal}

Situamos al pacientes lo más decúbito lateral posible, colocando de 3 a 4 puertos según necesidades. El primer puerto fue de $5 \mathrm{~mm}$ colocándose siempre de forma abierta a nivel umbilical para introducir la óptica y el resto, de 5 ó $3 \mathrm{~mm}$ dependiendo del tamaño del niño, situándose uno en la fosa iliaca correspondiente al lado afecto, otro en epigastrio a 1-2 $\mathrm{cm}$ por debajo del reborde de las costillas en lado contralateral y en ocasiones otro accesorio en el hipocondrio homolateral, a un través de dedo del arco costal. La pieza se extrajo por el ombligo, previa ampliación de la herida, en caso de riñones pequeños, o por el puerto de la fosa iliaca correspondiente, previa ampliación, en caso de riñones de gran tamaño.

\section{Vía retroperitoneal}

Posicionamos al paciente en decúbito lateral, colocando tres trócares, uno de $5 \mathrm{~mm}$ por debajo de la punta de la 12 costilla para la óptica, otro por encima de espina iliaca en línea media clavicular y otros en punto media de la línea de los músculos espinales. La pieza se extrajo ampliando la incisión del puerto de la óptica. En ambos abordajes el flujo de dióxido de carbono fue de 3,5 1/min y una presión entre $12-13 \mathrm{~mm}$ de Hg, dependiendo del tamaño y edad del niño. Para el control de los vasos del pedículo renal utilizamos de forma habitual la pinza Ligasure ${ }^{\circledR}$, aunque también utilizamos para la disección de los tejidos la "pinza electrocauterio de gancho" y/o el bisturí Harmónico. En algunas ocasiones usamos el láser para cortar y coagular el parénquima renal específicamente en resecciones parciales.

$\mathrm{El}$ análisis estadístico de los datos se realizó con el programa estadístico SPSS statistical software (SPSSCorp, Chicago, Illinois) ${ }^{\circledR}$.

La búsqueda bibliográfica se efectuó en las bases de datos MEDLINE, EMBASE Y COCHRANE, revisando las principales series de pacientes intervenidos de cirugía exerética renal por laparoscopia seleccionados por un investigador. Posteriormente todos fueron nuevamente revisados por un segundo investigador antes de ser añadidos al estudio. Sólo se incluyeron artículos con diez o más pacientes, series de patologías urológicas y series que comparaban la vía abierta con la laparoscopia. Excluimos aquellos que han incluido pacientes adultos o que no han hecho ninguna distinción en los resultados entre adultos y niños.

Todos los estudios fueron estratificados de acuerdo a la modificación de la Consulta Internacional sobre enfermedades urológicas de los niveles de evidencia del Oxford Centre for Evidence-based 
Medicine. Para la adecuada asignación de cada estudio consideramos el diseño del estudio (prospectivo/retrospectivo), número de pacientes incluidos, tipo de herramientas de cálculo, propiedades psicométricas (validez y fiabilidad) y tasa de respuesta. De acuerdo con esta clasificación todos los trabajos revisados tienen un nivel de evidencia III/B.

\section{RESULTADOS}

La edad de diagnóstico varió de 3 meses a 14 años, con una edad media de intervención de 3,6 años. Hubo 12 (21\%) pacientes con un peso igual o inferior a $10 \mathrm{Kg}$ y 7 (12,5\%) tenían menos de 1 año.

Los diagnósticos más frecuentes fueron el reflujo vésico-ureteral con 23 niños (41\%), 4 de ellos en sistemas dúplex, siguiéndole el riñón multiquístico con $17(30 \%)$ casos (Tabla 1). La indicación para intervenir fue la anulación funcional en 38 (67\%) casos; displasia multiquístico en 17 (30\%) casos y 1 (2\%) caso con quiste renal simple (Tabla 2).

Lo procedimientos utilizados en los 56 niños intervenidos han sido: 39 (70\%) nefrectomías, 13 (23\%) heminefrectomías, 3 (9\%) Nefroureterectomias y 1 (3\%) quiste renal simple (Tabla 3). En 51 niños (91\%) se utilizó la vía transperitoneal y en 5 (9\%) la vía retroperitoneal. El tiempo medio quirúrgico fue de $118 \pm 0,75 \mathrm{~min}$ para las nefrectomías, 192 $\pm 1,07 \mathrm{~min}$

Tabla 1. Diagnóstico Principal

\begin{tabular}{lc}
\hline Diagnóstico principal & n (\%) \\
\hline Reflujo vésico-ureteral & 23 (4 asoc. a Dupl.) (41) \\
R. Multiquístico & $17(30)$ \\
Ureterocele & $4(7)$ \\
$\begin{array}{l}\text { Duplicidad renal con } \\
\text { ecropia ureteral }\end{array}$ & $4(7)$ \\
Hidronefrosis & $4(7)$ \\
Megaureter & $2(4)$ \\
Quiste Renal & $1(2)$ \\
$\begin{array}{l}\text { Displasia renal } 2^{\text {a a Válvulas }} \\
\text { de Uretra Posterior }\end{array}$ & $1(2)$ \\
\hline
\end{tabular}

Tabla 2. Indicaciones de cirugía

\begin{tabular}{lc}
\hline Indicaciones de cirugia & $\mathbf{n}(\%)$ \\
\hline Anulación Funcional & $26(46)$ \\
Anulación Funcional en duplicidad & $12(21)$ \\
Anulación Funcional en R. Multiquístico & $17(30)$ \\
Tamaño de quiste renal simple & $1(2)$ \\
\hline
\end{tabular}

Tabla 3. Técnicas quirúrgicas laparoscópicas

\begin{tabular}{lc}
\hline Técnica quirúrgica & n (\%) \\
\hline Nefrectomías & $39(70)$ \\
Heminefrectomías & $13(23)$ \\
Nefro-ureterectomías & $3(5)$ \\
Quistectomía & $1(2)$ \\
\hline
\end{tabular}

para las nefrectomías parciales, $111 \pm 0,64 \mathrm{~min}$ para las nefroureterectomías y 240 min de tiempo total para la quistectomía. La estancia media hospitalaria fue de $3,18 \pm 0,63$ días para las nefrectomías, $5,91 \pm 2,25$ días para las nefrectomías parciales, $3 \pm 1$ días para las nefroureterectomías y 6 días de estancia completa para la quistectomía.

Las complicaciones encontradas ha sido de 8 (14\%), distribuyéndose de la siguiente forma:

\section{Complicaciones intraoperatorias}

Hubo 2 reconversiones (3\%), una por dificultades técnicas con la óptica y otra por mala visualización del campo quirúrgico a causa de sangrado.

\section{Complicaciones postoperatorias menores}

Tuvimos $3(5,5 \%)$ pacientes que presentaron cuadro febril el $2^{\circ}, 4^{\circ}$ y $9^{\circ}$ día del postoperatorio sin foco aparente, siendo tratados mediante cobertura antibiótica y observación de heridas y drenajes, resolviéndose a las 48 horas siguientes a su debut en todos los casos.

\section{Complicaciones postoperatorias mayores}

Ocurrieron en 3 niños (5,5\%), siendo un pseudoaneurisma de la pared de la arteria ilíaca izquierda tras nefrectomía por retroperitoneoscopia debido a la anulación $2^{\mathrm{a}}$ a ureterocele en un niño de 5 años en la que se utilizó la "pinza de gancho", que se descubrió al 4 mes del postoperatorio por dolor abdominal recurrente, practicándose ECO y visualizándolo, resolviéndose con drenaje del hematoma y sutura de la pared arterial mediante cirugía abierta, y dos urinomas perirenales. El primero de ellos sucedió tras heminefrectomía de pielón inferior que se detectó al 3 mes y precisó drenaje percutáneo con control ecográfico, siendo sustituido posteriormente, a las 2 semanas, por catéter ureteral doble J. El segundo ocurrió tras nefrectomía, dentro de los 15 primeros días del postoperatorio, en la que se seccionó el uréter con Ligasure ${ }^{\circledR}$, presentando fuga de orina por el muñón ureteral, que se resolvió reinterviniendo laparoscópicamente y cerrando el muñón ureteral con clip. 
Las pérdidas hemáticas intraoperatorias han sido mínimas $(<20 \mathrm{ml})$. En dos pacientes se colocaron drenajes que dieron un líquido serohemático mínimo.

En la revisión bibliográfica se han seleccionado 47 artículos; 5 (11\%) comparan cirugía laparoscópica/cirugía abierta en heminefrectomías y heminefroureterectomías, 3 (6\%) comparan cirugía laparos- cópica/cirugía abierta en nefrectomías y nefroureterectomias, 13 (28\%) series de nefrectomías y nefroureterectomías, 9 (19\%) series de heminefrectomías y heminefroureterectomías, 14 (30\%) series de cirugía laparoscópica y 3 (6\%) englobados en otros artículos. Las Tablas de 4-9 explican los objetivos de los distintos artículos, abordaje, resultados y complicaciones descritas.

Tabla 4. Series comparativas laparoscopia vs cirugía abierta

\begin{tabular}{|c|c|c|c|c|c|}
\hline Ref. & Objeto & $\mathbf{n}$ & Abord. & Resultados & Complicaciones \\
\hline $\begin{array}{l}\text { Robinson BC } \\
\text { et al. }{ }^{10}\end{array}$ & $\begin{array}{l}\text { Compara HL con } \\
\text { HA. }\end{array}$ & $\begin{array}{l}11 \mathrm{~L} \\
11 \mathrm{~A}\end{array}$ & Transp. & $\begin{array}{l}\text { Tm QL: } 200.4 \text { min y TmQA: } 113.5 \text { min. con > } \\
\text { coste L. P sig. < EM L, <Analgesia y mejor estética } \\
\text { L. P sig. en estancia y analgesia. } \\
\text { Sopesar pros y contras a la hora de decidir técnica. }\end{array}$ & $\begin{array}{l}\text { HL: } \\
\text { - } 1 \text { reconversión } \\
-2 \text { quistes en fosa renal } \\
\text { HA: } \\
1 \text { reintervención por uréter triple no } \\
\text { visto en c. previa. }\end{array}$ \\
\hline Lee RS et al. ${ }^{11}$ & $\begin{array}{l}\text { Compara HL con } \\
\text { HA. }\end{array}$ & $\begin{array}{l}14 \mathrm{~L} \\
14 \mathrm{~A}\end{array}$ & Retrop. & $\begin{array}{l}\text { Igual TmQ (p. ns), } 1.7 \text { dias hospitalización Laparo, } \\
4.7 \text { abierta. Menos analgesia intraoperatoria y postop } \\
\text { laparo. Resultados similares subanálisis en niños } \\
\text { < 2años. La HPL es segura y es ventajosa respecto } \\
\text { a CA. }\end{array}$ & $\begin{array}{l}\text { HL: } \\
1 \text { Urinoma (no intervención) }\end{array}$ \\
\hline Piaggio L et al. ${ }^{12}$ & $\begin{array}{l}\text { Compara HL con } \\
\text { HA. }\end{array}$ & $\begin{array}{l}14 \mathrm{~L} \\
20 \mathrm{~A}\end{array}$ & Transp. & $\begin{array}{l}\text { TmQA } 115 \text { min; TmQL } 180 \text { min. EM } 3 \text { dias abierta } \\
2 \text { días laparo. Necesarios más opioides para laparo. } \\
\text { Resultados parecidos a la abierta o mejores. Se } \\
\text { recomienda incluir la laparo en el arsenal terapéutico. }\end{array}$ & $\begin{array}{l}\text { HL: } \\
\text { - } 1 \text { hernia omental resuelta con } \\
\text { reposo en cama } \\
\text { - } 1 \text { un urinoma x HP de polo } \\
\text { sup-drenaje perc. } \\
\text { HA: } \\
\text { - } 1 \text { caso de sangrado ureteral, } \\
\text { - } 1 \text { caso de daño polo ureteral inf. }\end{array}$ \\
\hline $\begin{array}{l}\text { El Ghoneimi } \\
\text { et al. }{ }^{13}\end{array}$ & $\begin{array}{l}\text { Comparar NUPL } \\
\text { con NUPA. }\end{array}$ & $\begin{array}{l}15 \mathrm{la} \\
13 \mathrm{abt}\end{array}$ & Retrop. & $\begin{array}{l}\text { Tm QA } 146 \text { (50-180) min, Tm QL } 152 \text { (74-240) min. } \\
\text { EM 1.4 (1-3) abierta, 3,9 (3-5) laparo. Técnica segura } \\
\text { y efectiva. Con requerimiento de tiempo quirúrgico } \\
\text { similares a la cirugia abierta. } \\
\text { Ventaja disminuye la estancia. }\end{array}$ & $\begin{array}{l}\text { - } 1 \text { reconversión-desgarro peritoneal. } \\
\text { - } 1 \text { urinoma de una heminefrect } \\
\text { pielón inf. }\end{array}$ \\
\hline Chertin et al. ${ }^{14}$ & $\begin{array}{l}\text { Comparación HL } \\
\text { con HA. }\end{array}$ & $\begin{array}{l}10 \text { lap } \\
10 \mathrm{abt}\end{array}$ & Transp. & 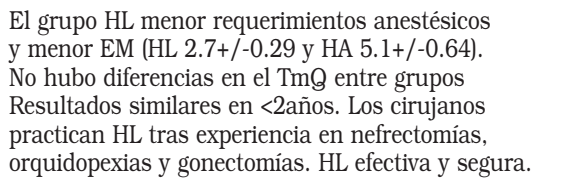 & $\begin{array}{l}\text { Grupo HL: } \\
\text { - } 1 \text { reconversión por daños de uréter } \\
\text { sano } \\
\text { Grupo HA: } \\
\text { - Débito urinario resuelto } \\
\text { espontáneamente. }\end{array}$ \\
\hline
\end{tabular}

HL: Heminefrectomías. NUPA: Nefroureterectomías parciales.

Tabla 5. Series comparativas laparoscopia vs cirugía abierta

\begin{tabular}{|c|c|c|c|c|c|}
\hline Ref. & Objeto & $\mathbf{n}$ & Abord. & Resultados & Complicaciones \\
\hline $\begin{array}{l}\text { Hamilton } \\
\text { et al. }{ }^{15}\end{array}$ & $\begin{array}{l}\text { Comparar NLT y una } \\
\text { NLR con NA. }\end{array}$ & $\begin{array}{l}10 \text { lap } \\
10 \mathrm{abt}\end{array}$ & $\begin{array}{l}\text { Trasp } \\
\text { Retrop }\end{array}$ & $\begin{array}{l}1 \text { Retrop. TmQL } 175,6 \mathrm{~min} \text {; Tm } \mathrm{QA} \text { 120,2 min. EM } \\
\text { laparo } 22,5 \text { horas; abierta } 41,3 \text { h. P sig. } \\
\text { Es un método seguro, se requieren más estudios. }\end{array}$ & $\begin{array}{l}1 \text { hematoma retroperitoneal tras una } \\
\text { heminefrectomía, no necesitó intervención. } \\
1 \text { daño serosa colon, tratado sin secuelas. }\end{array}$ \\
\hline Ku et al. ${ }^{16}$ & $\begin{array}{l}\text { Comparar NL } \\
\text { (retroperitoneal) con } \\
\text { NA. }\end{array}$ & $\begin{array}{l}10 \text { lap } \\
13 \mathrm{abt}\end{array}$ & Retrop & $\begin{array}{l}\text { EM A } 4 \text { días (3-14), L 2,5 (2-6) días. Tm QL } 150 \text { min, } \\
\text { Tm QA } 145 \text { min. Sangrado laparo } 20 \mathrm{ml} \text { us abierta } \\
20 \text { ml. La retrop. indicada en patologia benigna } \\
\text { con minima morbilidad, mejor resultados cosméticos } \\
\text { y corta estancia hospitalaria. Puede ser superior a la } \\
\text { cirugia abierta. }\end{array}$ & $\begin{array}{l}\text { No reconversiones, no complicaciones } \\
\text { mayores. Complicación de una herida } \\
\text { en el grupo de cirugía abierta. }\end{array}$ \\
\hline Sekaran et al. ${ }^{17}$ & Compara NL con NA. & $\begin{array}{l}26 \text { lap } \\
22 \mathrm{abt}\end{array}$ & $\begin{array}{l}\text { Trans } \\
\text { Retrop }\end{array}$ & $\begin{array}{l}14 \text { transp, } 12 \text { retrop. TmQ abt } 62 \text { min. EM } 4 \text { dias abt, } \\
2 \text { dias Transp., } 1 \text { dia retrop. Reducción del } 50 \% \text { en } \\
\text { dosis de analgésicos utilizados lap frente a abt. Menor } \\
\text { analgesia para retrop que para Transp. Menor EM y } \\
\text { analgesia para niños operados por lap. }\end{array}$ & $\begin{array}{l}3 \text { conversiones: } \\
\text { - Fallo del equipo } \\
\text { - Sangrado } \\
\text { - Obesidad asociada con cifoescoliosis }\end{array}$ \\
\hline
\end{tabular}


Tabla 6. Series

\begin{tabular}{|c|c|c|c|c|c|}
\hline Ref. & Objeto & $\mathbf{n}$ & Abord. & Resultados & Complicaciones \\
\hline Valla et al. ${ }^{18}$ & 18 casos de NL. & 18 lap & Retrop. & $\begin{array}{l}\text { TmQ 35-210 min ( } 106 \text { media). Técnica segura y } \\
\text { efectiva en manos expertas. }\end{array}$ & $\begin{array}{l}1 \text { reconversión } \\
\text { No complicaciones mayores. }\end{array}$ \\
\hline $\begin{array}{l}\text { Kobashi KC } \\
\text { et al. }{ }^{19}\end{array}$ & $\begin{array}{l}\text { Resultados en NL } \\
\text { y NUL. }\end{array}$ & 20 lap & Retrop. & $\begin{array}{l}15 \text { NL, } 5 \text { NUL. TmQ } 1 \text { h } 42 \text { min. Todos alta inmediata } \\
\text { con asistencia domiciliaria. Buenos resultados y } \\
\text { satisfacción de pacientes. Utiliza cuestionario de } \\
\text { satisfacción aplicado a los padres. }\end{array}$ & $\begin{array}{l}\text { - } 1 \text { reconversión por falta de visión por } \\
\text { sangrado. } \\
\text { - Fiebre desconocida (hospitalización } 3 \\
\text { dias). } \\
\text { - Fuga por muñón ureteral ( reintervención). }\end{array}$ \\
\hline Davies et al. ${ }^{20}$ & $\begin{array}{l}\text { Experiencia NL y } \\
\text { NUL. }\end{array}$ & 24 lap & Transp. & $\begin{array}{l}12 \text { NUL. TmQ } 85 \text { (40-160) min. EM 2(1-4) días. } \\
\text { Procedimiento seguro y útil en nefroureterectomía. }\end{array}$ & \\
\hline $\begin{array}{l}\text { Yao D and } \\
\text { Poppas D }\end{array}$ & $\begin{array}{l}\text { Experiencia en NL, } \\
\text { NUL y HL. }\end{array}$ & 26 lap & Transp. & $\begin{array}{l}14 \text { NL, } 6 \text { NUL y } 6 \text { HL. TQ } 165 \text { min. Sangrado } 5 \mathrm{cc} \text {. } \\
\text { EM } 3 \text { días, alta el mismo día y } 17,3,1 \text { y } 2=1,2,4 \\
\text { y } 5 \text { días respectivamente. } \\
\text { La técnica laparoscópica está indicada en patologías } \\
\text { benignas, con baja morbilidad, pocas molestias, } \\
\text { mejoras cosméticas y corta estancia. }\end{array}$ & \\
\hline Dronov et al. ${ }^{22}$ & $\begin{array}{l}\text { Experiencia en NL y } \\
\text { NUL. }\end{array}$ & 72 lap & Transp. & $\begin{array}{l}\text { TmQ } 30-150 \text { min (media } 45 \text { min) Sangrado }<\text { de } \\
50-60 \text { ml. Procedimiento poco traumático, seguro } \\
\text { y efectivo en niños de cualquier edad en diferentes } \\
\text { patologías renales. }\end{array}$ & \\
\hline Ku et al. ${ }^{23}$ & $\begin{array}{l}\text { Evalúa experiencia } \\
\text { del cirujano. } \\
\text { Dos grupos a partir } \\
\text { del punto medio de } \\
\text { corte. Comparación } \\
\text { entre NL y NUL. }\end{array}$ & 20 lap & $\begin{array}{l}\text { Transp. } \\
\text { Retrop. }\end{array}$ & $\begin{array}{l}14 \text { transp, } 6 \text { retrop. TmQ en los primeros } 10 \text { casos } \\
181 \text { min, y } 125 \text { min en los segundos } 10 \text { casos. } \\
\text { Sangrado y tolerancia menor en el segundo grupo. } \\
\text { EM } 5,4 \text { días en los primeros } 10 \text { casos y } 2,5 \text { en los } \\
\text { segundos } 10 \text { casos. Disminuye tiempo quirúrgico } \\
\text { cuando la experiencia del cirujano excede los } 10 \text { casos. }\end{array}$ & \\
\hline Mahomed et al. ${ }^{24}$ & $\begin{array}{l}\text { Estudio } \\
\text { prospectivo } \\
\text { multicéntrico NL } \\
\text { y NUL. }\end{array}$ & 30 lap & Transp. & $\begin{array}{l}\text { Edad media 4,43 (3-15) años. TmQ 93+/-30 min. } \\
\text { EM } 1 \text { (0-16) días. Seguimiento } 2,88 \text { años. } 1 / 3 \text { de los } \\
\text { pacientes requirieron morfina en el postoperatorio } \\
\text { inmediato. Técnica segura sin complicaciones } \\
\text { que se considera una alternativa en esta cirugía. }\end{array}$ & $\begin{array}{l}\text { Intraoperatorias: } \\
\text { - } 2 \text { sangrado } \\
\text { - } 1 \text { dificultad localización } \\
\text { - } 1 \text { dificultad extracción } \\
\text { - } 2 \text { retractor hepático } \\
\text { Postoperatorias: } \\
\text { - } 2 \text { ITUs por muñón ureteral remanente } \\
\text { que requirió cirugía }\end{array}$ \\
\hline Jeong BC et al. ${ }^{25}$ & $\begin{array}{l}\text { Eficacia NL por } \\
\text { uréter ectópico } \\
\text { drenando riñón } \\
\text { displásico. }\end{array}$ & 16 lap & Transp. & $\begin{array}{l}\text { TmQ } 109 \text { (40-155) min. Sangrado minimo. } \\
\text { EM 2,6 (2-4) días.Técnica segura y efectiva, } \\
\text { puede realizarse con éxito incluso si las técnicas } \\
\text { de imagen previas no han visualizado correctamente } \\
\text { el riñón displásico. }\end{array}$ & $\begin{array}{l}\text { Intraoperatorias } \\
\text { - Daño intestinal con trócar de Hasson. }\end{array}$ \\
\hline Gundeti et al. ${ }^{26}$ & $\begin{array}{l}\text { Nefrectomia bilateral } \\
\text { sincrónica } \\
\text { retroperitoneal y } \\
\text { diálisis peritoneal } \\
\text { inmediata en las } \\
24 \text { h. siguientes } \\
\text { a la cirugía. }\end{array}$ & 20 lap & Retrop. & $\begin{array}{l}\text { TmQ } 160 \text { ( } 110-180 \text { min). No requeridas transfusiones. } \\
\text { Diálisis peritoneal en las } 24 \text { h siguientes en } 15 \text { enfermos } \\
\text { en } 2 \text { en } 48 \text { h siguientes y en uno hemodiálisis transitoria. }\end{array}$ & $\begin{array}{l}\text { Conversión unilateral en un caso por } \\
\text { desgarro peritoneal. }\end{array}$ \\
\hline Jesch et al. ${ }^{27}$ & $\begin{array}{l}\text { NUL en niños } \\
\text { menores de } 1 \text { año } \\
\text { en relación con el } \\
\text { tamaño renal. }\end{array}$ & 40 lap & Transp. & $\begin{array}{l}19 \text { niños < } 1 \text { año. TmQ } 133(60-240) \text { min. } 126(60-240) \\
\text { min en niños < } 1 \text { año. Efectividad de la NUL en } \\
<1 \text { año de edad y no viene afecto por el tamaño } \\
\text { del riñón o patologías renales subyacentes. }\end{array}$ & 3 conversiones por falta de visibilidad. \\
\hline Duarte et al. ${ }^{28}$ & $\begin{array}{l}\text { NL para tumor de } \\
\text { Wilms en niños } \\
\text { tratados con } \\
\text { quimioterapia } \\
\text { neoadyuvante. }\end{array}$ & 16 lap & Transp. & $\begin{array}{l}\text { Media tamaño tumoral } 9.7 \times 5.4 \mathrm{~cm} .8 \text { niños edad } \\
\text { media } 44.3 \text { meses. Un caso adherido al hígado. } \\
\text { Linfadenectomía tras NL } 3-12 \text { nódulos. } \\
\text { TmQ } 135 \text { (120-180) min. Sangrado } 30-50 \mathrm{ml} \text {. } \\
\text { Alta en 2-3 dias. Seguimiento 5-23 meses sin } \\
\text { recurrencias. NL tras Quimioterapia es segura en casos } \\
\text { seleccionados. Son necesarios resultados a largo plazo } \\
\text { y más pacientes para comparar con la cirugía abierta. }\end{array}$ & \\
\hline Lam et al. ${ }^{29}$ & $\begin{array}{l}\text { Determinar } \\
\text { beneficios NL. }\end{array}$ & 32 lap & $\begin{array}{l}\text { Transp. } \\
\text { Retrop. }\end{array}$ & $\begin{array}{l}22 \text { transp, } 10 \text { retrop. TmQ } 65 \text { min retrop, } 95 \text { min } \\
\text { Transp. Menos requerimientos anestésicos en retrop. } \\
\text { La mayoría de los niños alta en } 2 \text { días. } \\
\text { La retrop parece ser segura y más rápida en niños } \\
\text { con efectos fisiológicos reducidos y menos dolor que } \\
\text { el abordaje Transp. }\end{array}$ & $\begin{array}{l}5 \text { conversiones: } \\
\text { - Sangrado } \\
\text { - Dificultad de disección } \\
\text { - Daño de la óptica } \\
\text { - Adherencias por una pielonefritis } \\
\text { xantogranulomatosa no diagnosticada } \\
\text { - Gran cantidad de grasa retroperitoneal } \\
\text { en un niño con escoliosis }\end{array}$ \\
\hline Steven et al. ${ }^{30}$ & $\begin{array}{l}\text { Intervenidos por } \\
\text { laparoscopia por } \\
\text { Riñón displásico } \\
\text { unilateral. }\end{array}$ & 13 lap & Transp. & $\begin{array}{l}\mathrm{TmQ} 86(50-135) \text { min. EM } 27(5-51) \mathrm{h} \text {. NL es un } \\
\text { procedimiento seguro y reproducible para el tratamiento } \\
\text { de esta patologia }\end{array}$ & \\
\hline
\end{tabular}

NL: Nefrectomías. NUL: Nefroureterectomias. 
Tabla 7. Series

\begin{tabular}{|c|c|c|c|c|c|}
\hline Ref. & Objeto & $\mathbf{n}$ & Abord. & Resultados & Complicaciones \\
\hline $\begin{array}{l}\text { Janestschek } \\
\text { et al. }{ }^{31}\end{array}$ & $\begin{array}{l}\text { Mostrar su } \\
\text { experiencia en HL. }\end{array}$ & 14 lap & Transp. & $\begin{array}{l}\text { TmQ } 222 \text { min HL(1); } 427 \text { min HUL(2). EM (1) 4,4 días; } \\
\text { (2) 7,5 dias. Sangrado minimo. Técnica segura, minimo } \\
\text { sangrado, baja morbilidad, baja tasa de complicaciones. } \\
\text { Debe hacerse en centros especializados. }\end{array}$ & \\
\hline Borer et al..$^{32}$ & $\begin{array}{l}\text { Pacientes } \mathrm{NL} \\
\text { retroperitoneal } \\
\text { usando trócares } \\
\text { de } 2 \mathrm{~mm} \text {. }\end{array}$ & 14 lap & Retrop. & $\begin{array}{l}\text { Edad } 3 \text { meses a } 13 \text { años. TmQ } 142 \text { min, sangrado } 15 \mathrm{ml} \text {, } \\
\text { EM } 2 \text { días media. Modificaciones en el abordaje } \\
\text { laparoscópico trócares de } 2 \mathrm{~mm} \text { y posición prono, } \\
\text { mejoran la eficacia y seguridad de esta técnica en niños. }\end{array}$ & \\
\hline York et al. ${ }^{33}$ & $\begin{array}{l}\text { Experiencia inicial } \\
\text { en NL. }\end{array}$ & 11 lap & Transp. & $\begin{array}{l}\text { TmQ } 163 \mathrm{~min}(90-420) \text {. Sangrado }<10-150 \mathrm{ml} \text { (media } 45) \text {. } \\
7 \text { pac se fueron al segundo día y } 2 \text { al } 5^{\circ} \text { día. } \\
\text { Requiere un buen aprendizaje. }\end{array}$ & $\begin{array}{l}\text { Ileo paralítico en } 5 \text { pac que retrasó } \\
\text { el alta }\end{array}$ \\
\hline Horowitz et al. ${ }^{34}$ & $\begin{array}{l}\text { Presentar la serie de } \\
\text { HL de polo superior. }\end{array}$ & 14 lap & Transp. & $\begin{array}{l}\text { TmQ } 100 \text { min. Sangrado <30 ml. EM de } 2,6 \text { días. } \\
\text { La HL transperitoneal es un método preferible a la abierta: } \\
\text { por el aumento en la visibilidad, pérdidas mínimas de sangre. }\end{array}$ & \\
\hline Valla et al. ${ }^{35}$ & $\begin{array}{l}24 \text { HL de pielón } \\
\text { superior por } \\
\text { retroperitoneoscopia. }\end{array}$ & 24 lap & Retrop. & $\begin{array}{l}\text { Tiempo quirúrgico } 2 \text { horas } 40 \text { min. EM } 3,4 \text { dias. } \\
\text { Los resultados son comparables a la cirugía abierta } \\
\text { convencional. Ventaja menos cicatrices. }\end{array}$ & $\begin{array}{l}\text { - } 3(12,5 \%) \text { reconversión. } \\
\text { - } 9(37 \%) \text { intraoperatorias: Desgarro } \\
2^{\text {a }} \text { parte del duodeno. } 2 \text { casos de } \\
\text { apertura del cáliz del pielón inf. } 5 \text { casos } \\
\text { de perforación peritoneal. } \\
\text { - } 5(20 \%) \text { postoperatorias: } 4 \text { colecciones } \\
\text { moderadas-observación. } 1 \text { colección } \\
\text { grande-drenaje y catéter doble j con } \\
\text { anestesia. }\end{array}$ \\
\hline Castellan et al. ${ }^{36}$ & $\begin{array}{l}\text { Mostrar la experiencia } \\
\text { en cia laparoscópica } \\
\text { vía retroperitoneal y } \\
\text { transperitoneal y } \\
\text { ver la diferencia entre } \\
\text { los abordajes. }\end{array}$ & 48 lap & $\begin{array}{l}\text { Transp. } \\
\text { Retrop. }\end{array}$ & $\begin{array}{l}32 \text { pac (67\%) Transp.; } 16 \text { pac (33\%) retrop. Ambas vías } \\
\text { pueden ser utilizadas para enfermedad benigna en niños } \\
\text { con mínima morbilidad, mejoras cosméticas y corta } \\
\text { estancia hospitalaria. Las complicaciones no dependen } \\
\text { de la vía de abordaje, pero sí de la edad del paciente. }\end{array}$ & $\begin{array}{l}\text { Retrop: } \\
\text { - } 2 \text { conversiones, } 1 \text { a heminefrectomía } \\
\text { abierta y otra a abordaje laparoscópico } \\
\text { transperitoneal. } \\
2 \text { pc con complicaciones: } \\
\text { - } 1 \text { fistula urinaria con resolución espont. } \\
\text { - } 1 \text { urinoma-tto conserv } \\
\text { Transp.: } \\
\text { - } 1 \text { pneumotorax tto con tubo endotorácico. } \\
\text { - } 1 \text { infección urinaria recurrente-tto } \\
\text { excisión de remanente ureteral } \\
\text { - } 1 \text { Hta. } \\
4 \text { de las } 5 \text { compl se vieron en }<1 \text { año. }\end{array}$ \\
\hline Wallis et al. ${ }^{37}$ & $\begin{array}{l}\text { Análisis de intervenidos } \\
\text { mediante HL } \\
\text { retroperitoneal. }\end{array}$ & 19 lap & Retrop. & $\begin{array}{l}\text { Técnica relativamente segura, con baja morbilidad. } \\
\text { Complicaciones serias pueden suceder de forma } \\
\text { tardía. }\end{array}$ & $\begin{array}{l}\text { - } 4 \text { reconversiones por mala exposición. } \\
\text { - } 3 \text { pérdidas urinarias resueltas con } \\
\text { sonda Foley. } \\
\text { - } 2 \text { colecciones retroperitoneales. } \\
\text { - } 1 \text { fiebre postoperatoria el 3er día. } \\
\text { - } 1 \text { hta } 32 \text { postoperatorio. }\end{array}$ \\
\hline Yucel et al. ${ }^{38}$ & $\begin{array}{l}\text { Evolución de la } \\
\text { experiencia en } \\
\text { los primeros } \\
6 \text { años de cirugía } \\
\text { laparoscópica. }\end{array}$ & 39 lap & $\begin{array}{l}\text { Transp. } \\
\text { Retrop. }\end{array}$ & $\begin{array}{l}26 \text { NL: } 5 \text { transp, } 21 \text { retrop. TmQ } 205 \text { min (110-230min). } \\
\text { Sangrado 21,5 cc. } \\
\text { 14 HL: } 9 \text { transp, } 5 \text { retrop. TmQ } 291 \text { min (140-420min), } \\
\text { Sangrado 65,3 cc. } \\
\text { El tiempo operatorio mejora a los } 4 \text { años de experiencia } \\
\text { significativamente en NL y no sig en HL. Sangrado mejora } \\
\text { sig en NL y HL. Los resultados son una guía de los } \\
\text { inicios de la laparoscopia. }\end{array}$ & $\begin{array}{l}\text { Conversión en HL por poca visibilidad. } \\
2 \text { Urinomas-conservador. }\end{array}$ \\
\hline Déne et al. ${ }^{39}$ & $\begin{array}{l}\text { Resultados de HL } \\
\text { de polo sup en niños } \\
\text { con doble sistema. }\end{array}$ & 19 lap & Transp. & $\begin{array}{l}\text { TmQr } 147 \mathrm{~min}(110-180 \mathrm{~min}) \text {. Seguimiento } 57.1 \text { meses. } \\
\text { Sangrado }<50 \mathrm{ml} \text {. }\end{array}$ & $\begin{array}{l}\text { ITU en } 5(29 \%) \text { - conservador } \\
\text { Empiema } 3 \text { niños - cirugía. }\end{array}$ \\
\hline
\end{tabular}

HL: Heminefrectomías. HUL: Heminefroureterectomías.

Hemos contabilizado 347 nefrectomías/nefroureterectomías laparoscópicas con buenos resultados en lo referente a estancias hospitalarias, sangrados y tiempo quirúrgico ${ }^{18-29}$. En este grupo de artículos como complicaciones podemos señalar que el grupo de Kobashi ${ }^{19}$ refiere un caso de fuga por muñón ureteral tras nefrectomía que requirió reintervención y el grupo de
Mahomed ${ }^{24}$ reseña 6 complicaciones intraoperatorias y 2 postoperatorias. En este grupo han habido 16 reconversiones a cirugía abierta ${ }^{18,19,24,27,29}$, la mayoría debidas a sangrado. En el grupo de artículos de las heminefrectomias/hemiureterectomías laparoscópicas se han descrito más complicaciones que en el grupo de las nefrectomías/nefroureterectomías laparoscópicas ${ }^{31-39}$. 
Tabla 8. Series de cirugía laparoscópica

\begin{tabular}{|c|c|c|c|c|c|}
\hline Ref. & Objeto & $\mathbf{n}$ & Abord. & Resultados & Complicaciones \\
\hline Valla et al. ${ }^{40}$ & $\begin{array}{l}\text { Análisis casos } \\
\text { operados por } \\
\text { laparoscópica. }\end{array}$ & 88 lap & Retrop. & $\begin{array}{l}50 \text { nefrectomías, } 5 \text { quistes renales, } 3 \text { pielolitotomías, } \\
2 \text { obstrucciones pieloureterales, } 2 \text { adrenalectomías, } \\
1 \text { uréter retrocavo, } 25 \text { varicoceles. TmQ } 96 \text { min (35-210), } \\
\text { EM } 2 \text { días. El abordaje retroperitoneal requiere } \\
\text { entrenamiento y requiere precaución en los niños por el } \\
\text { reducido espacio y la fragilidad del peritoneo. Las ventajas } \\
\text { parecen suficientes para recomendar esta técnica. }\end{array}$ & $\begin{array}{l}7 \text { Reconversiones }(8 \%) \\
\text { Intraoperatorias: } \\
\text { - perforación duodenal } \\
\text { - quemadura ureteral } \\
\text { - quemadura ureteral } \\
\text { - perforación peritoneal } \\
\text { Postoperatorias: } \\
\text { - } 2 \text { urinomas tras heminefrectomias } \\
\text { - } 1 \text { hidrocele } \\
\text { - } 1 \text { varicocele recidivado-IQ posterior } \\
\text { - } 1 \text { recurrencia de litiasis-IQ posterior } \\
\text { Reintervención daño ureteral. }\end{array}$ \\
\hline $\begin{array}{l}\text { El-Ghoenimi } \\
\text { et al. }{ }^{41}\end{array}$ & $\begin{array}{l}\text { Resultados cirugía } \\
\text { renal laparoscopica } \\
\text { retroperitoneal } \\
\text { (nefrectomias, } \\
\text { heminefrectomias, } \\
\text { quistectomias, } \\
\text { pielitotomia) }\end{array}$ & 42 lap & Retrop. & $\begin{array}{l}31 \text { nefrectomias, } 8 \text { heminefrectomías, } 2 \text { quistectomías, } \\
\text { y } 1 \text { pielolitotomía. TmQ } 104 \text { min nefrectomías; } 153 \text { min } \\
\text { nefroureterectomias; } 135 \text { min pielolitotomía. } \\
\text { Aumento de las indicaciones de } 1 \text { cir. renal laparoscópica } \\
\text { econ el tiempo, según la experiencia del equipo. El abordaje } \\
\text { retroperitoneal es una técnica comparable } \\
\text { a la cirugía renal abierta. }\end{array}$ & $\begin{array}{l}\text { Nefrectomias: } 8 \text { desgarros peritoneales- } \\
\text { sin precisar tto. } \\
\text { Heminefrectomias: } 2 \text { reconversiónes por } \\
\text { no identificar los vasos polares, en } 1 \text { de } \\
\text { ellas se vio una perforación duodenal. } \\
\text { Desgarro peritoneal en } 4 \text { casos. } \\
\text { Pielolitotomía: Recurrencia al año, } \\
\text { reintervención por cia abierta. }\end{array}$ \\
\hline $\begin{array}{l}\text { Luque Mialdea } \\
\text { et al. }{ }^{42}\end{array}$ & $\begin{array}{l}\text { Establecer las } \\
\text { patologias urológicas } \\
\text { subsidiarias de } \\
\text { cirugía video } \\
\text { asistida frente a la } \\
\text { cirugía convencional. }\end{array}$ & 106 lap & $\begin{array}{l}\text { Retrop. } \\
\text { Transp. }\end{array}$ & $\begin{array}{l}18 \text { Nefrectomías (retrop), } 2 \text { quiste renal, } 4 \text { linfocele gigante } \\
\text { tras transplante; resto procesos menores. Efectividad del } \\
94,2 \% \text { y } 100 \% \text { de los procedimientos diagnósticos y } \\
\text { terapéuticos. Morbilidad intraoperatoria } 2,8 \% \text {. EM } 1,4 \text { días. } \\
\text { Ciertos procedimientos urológicos en pediatría deben ser } \\
\text { considerados indicaciones de laparoscopia. }\end{array}$ & $\begin{array}{l}\text { Conversiones 2: perforación peritoneal } \\
\text { (nefrectomia) y sangrado en biopsia renal } \\
\text { retrop. }\end{array}$ \\
\hline Seibold $\mathrm{J}$ et al. ${ }^{43}$ & $\begin{array}{l}\text { Indicaciones } \\
\text { diagnósticas y } \\
\text { terapéuticas en cirugía } \\
\text { laparoscópica } \\
\text { pediátrica. }\end{array}$ & 86 lap & Transp. & $\begin{array}{l}23 \text { criptorquidias, } 37 \text { varicoceles, } 4 \text { hidroceles, } \\
6 \text { reimplantes vesicoureterales, } 3 \text { nefroureterectomías, } \\
9 \text { heminefrectomias y } 2 \text { divertículos vesicales. Es un } \\
\text { procedimiento adecuado para la criptorquidia y varicoceles, } \\
\text { que requiere una mayor demanda técnica en cirugía renal, } \\
\text { aconsejable en centros especializados. }\end{array}$ & 1 complicación \\
\hline Shanberg et al. ${ }^{44}$ & $\begin{array}{l}\text { Establecer el uso de de } \\
\text { la retroperitoneoscopia } \\
\text { para la nefrectomía y } \\
\text { adrenalectomía. }\end{array}$ & 48 lap & Retrop. & $\begin{array}{l}22 \text { nefrectomias, } 15 \text { nefroureterectomias, } 6 \text { biopsias renales, } \\
2 \text { quistectomias y } 3 \text { adrenelectomías. TmQ } 75 \text { min, y } 115 \text { min } \\
\text { (adrenalectomía). } 11 \text { pacientes eran }<1 \text { año. Es seguro y } \\
\text { rápido. Además ofrece ventajas cosméticas y una más rápida } \\
\text { recuperación. }\end{array}$ & $\begin{array}{l}2 \text { conversiones, una adrenal (por no } \\
\text { control de la vena) y una nefrectomía } \\
\text { (por sangrado). } \\
1 \text { desgarro peritoneal. }\end{array}$ \\
\hline Ehrlich et al. ${ }^{45}$ & $\begin{array}{l}\text { Experiencia } \\
\text { laparoscopica }\end{array}$ & 17 lap & Retrop. & $\begin{array}{l}10 \text { nefrectomías, } 4 \text { nefroureterectomias, } 2 \text { nefrectomías } \\
\text { parciales y un quiste gigante. TmQ } 2 \text { horas y } 15 \mathrm{~min} \\
(1.50-2.45) \text {. EM } 23 \text { horas, hasta } 36 \mathrm{hrs} \text { en } 2 \text { pac. La } \\
\text { laparoscopia ofrece ventajas a pesar del espacio pequeño de } \\
\text { trabajo, menor estancia, menos dolor, mejoras cosméticas } \\
\text { y pronta recuperación. }\end{array}$ & \\
\hline Tadini et al. ${ }^{46}$ & $\begin{array}{l}\text { Experiencia en } \\
\text { cirugía laparoscópica. }\end{array}$ & 32 lap & $\begin{array}{l}\text { Retrop. } \\
\text { Transp. }\end{array}$ & $\begin{array}{l}27 \text { nefrect retrop, } 1 \text { nefrecto bilat Transp., } 2 \text { quistes renales, } \\
1 \text { biopsia renal. EM } 2 \text { días. Seguimiento medio } 30 \text { (6-64) meses. } \\
\text { La cirugia laparoscópica minima morbilidad, disconfort, } \\
\text { buenos resultados cosméticos y estancia corta. }\end{array}$ & $\begin{array}{l}\text { Intraoperatorias } \\
-6 \text { laceraciones peritoneales. } \\
\text { Postoperatorias } \\
\text { - sangrado retroperitoneal-trasfusión. }\end{array}$ \\
\hline $\begin{array}{l}\text { Fahlenkamp } \\
\text { et al. }{ }^{47}\end{array}$ & $\begin{array}{l}\text { Experiencia en } \\
\text { cirugía } \\
\text { laparoscópica en } \\
\text { edad pediátrica. }\end{array}$ & 219 lap & Transp. & $\begin{array}{l}\text { TmQ: Testículos no palpables } 10-30 \text { min. Varicocele } 15-30 \text { min. } \\
\text { Nefrectomía } 80-150 \text { min. Exéresis de uraco o linfocele } \\
30-70 \text { min. Buena aplicación para los testículos no } \\
\text { palpables, varicocele y linfocele. Técnica difícil en } \\
\text { nefrectomia. Coste elevado respecto a la cirugia abierta. } \\
\text { Papel cada vez más importante. }\end{array}$ & \\
\hline $\begin{array}{l}\text { Urbanowicz } \\
\text { et al. }{ }^{48}\end{array}$ & $\begin{array}{l}\text { Experiencia primeros } \\
\text { casos } \\
\text { retroperitoneoscopia } \\
\text { en prono-posición. }\end{array}$ & 12 lap & Retrop. & $\begin{array}{l}\text { Nefrectomía, Nefroureterectomía o heminefroureterectomía. } \\
\text { Edad media 3,5 años. Causa obstrucción, reflujo o displasia } \\
\text { renal multiquística. }\end{array}$ & \\
\hline Gundeti et al.49 & $\begin{array}{l}\text { Experiencia inicial } \\
\text { en cirugía } \\
\text { laparoscópica. }\end{array}$ & 100 lap & $\begin{array}{l}\text { Transp. } \\
\text { Retrop. }\end{array}$ & $\begin{array}{l}51 \text { transp y } 49 \text { retrop. Nefrectomias. TmQr Retrop } 96 \text { min; } \\
\text { Transp. } 112 \text { min (incluido preparación del equipo y sutura } \\
\text { heridas). } 5 \text { niños nefrectomias sincrónica bilateral. } \\
\text { EM Trans } 2 \text { dias y Retrop } 1 \text { día (no signif). No dif en analgesia. } \\
\text { La retrop se puede realizar con } 2 \text { puertos y es adecuada para } \\
\text { niños con enf renal terminal que precisan nefrectomía bilateral. }\end{array}$ & $\begin{array}{l}\text { Retrop: } \\
\text { - Abceso intraperitoneal-conservador } \\
\text { - Urinoma-conservador } \\
\text { - Infección herida del puerto-conservador. } \\
\text { Transp.: } \\
\text { - Infección resp-conservador } \\
\text { - Derrame pleural-Aspiración }\end{array}$ \\
\hline $\begin{array}{l}\text { Mulholland } \\
\text { et al. }{ }^{50}\end{array}$ & $\begin{array}{l}\text { Experiencia } \\
\text { laparoscópica en } \\
\text { niños de menos } \\
\text { de } 10 \mathrm{~kg} \text { de peso. }\end{array}$ & 17 lap & Transp. & $\begin{array}{l}12 \text { nefrectomias, } 3 \text { nefroureterectomías y } 2 \text { heminefrectomías. } \\
\text { TmQ } 138 \text { (77-229) min. EM } 23 \text { horas o menos en todos } \\
\text { menos en dos. Procedimientos mayores laparoscópicos } \\
\text { pueden ser realizados en niños < } 10 \mathrm{~kg} \text { con baja morbilidad y } \\
\text { recuperación rápida. }\end{array}$ & Daño diafragmático. \\
\hline
\end{tabular}


Tabla 8. Series de cirugía laparoscópica (Continuación)

\begin{tabular}{|c|c|c|c|c|c|}
\hline Ref. & Objeto & $\mathbf{n}$ & Abord. & Resultados & Complicaciones \\
\hline Farhat et al. ${ }^{51}$ & $\begin{array}{l}\text { Establecer la } \\
\text { seguridad en el } \\
\text { aprendizaje de } \\
\text { urólogos pediatras } \\
\text { en retroperito- } \\
\text { neoscopia. }\end{array}$ & 36 lap & Retrop. & $\begin{array}{l}4 \text { cirujanos con diferente experiencia, realizan procesos } \\
\text { laparoscópicos con ayuda de un mentor durante } 10 \text { meses: } \\
13 \text { HNL, } 7 \text { pieloplastias y } 1 \text { pielotomia. TmQ NL } 130 \\
\text { (75-170) min, HNL 188(75-140) min; Pieloplastia } \\
240 \text { (195-300) min. Tras } 10 \text { meses: } 10 \mathrm{NL} \text {, 1HNL. } \\
\text { TmQ NL } 180 \text { (135-240)min; HNL 150min El aprendizaje } \\
\text { con el mentor permite familiarizarse con las habilidades } \\
\text { básicas, pero no sustituye el entrenamiento ordinario } \\
\text { laparoscópico adquirido durante un fellowship o residencia. }\end{array}$ & $\begin{array}{l}5 \text { conversiones: (con mentor) } \\
\text { - } 1 \text { NL Adherencias por cirugía previa } \\
\text { - } 1 \text { HNL Insuflación intraperitoneal } \\
\text { de CO2 } \\
\text { - } 3 \text { pieloplastias por difultad técnica } \\
\text { de la anastomosis } \\
1 \text { hematoma retroperitoneal- drenaje } \\
\text { percutáneo. } \\
5 \text { conversiones: (sin mentor) } \\
\text { - } 2 \text { NL insuflación peritoneal } \\
\text { - } 2 \text { NL dificultad técnica } \\
\text { - } 1 \text { pieloplastia dificultad técnica }\end{array}$ \\
\hline $\begin{array}{l}\text { Capolicchio } \\
\text { et al. }{ }^{52}\end{array}$ & $\begin{array}{l}\text { Acceso modificado } \\
\text { para cirugía } \\
\text { retroperitoneal } \\
\text { en niños. }\end{array}$ & 18 lap & Retrop. & $\begin{array}{l}\text { Modif. Posición trócares. } 12 \text { nefrectomias, } 3 \text { pieloplastias, } \\
1 \text { nefrectomía parcial, } 1 \text { nefroureterectomía y } 1 \text { decorticación } \\
\text { de un quiste parapiélico. TmQ } 178 \text { min (120-240 min). } \\
\text { EM 2,6 dias (1 a 9). }\end{array}$ & $\begin{array}{l}\text { - } 1 \text { conversión por dificultad en la } \\
\text { anastomosis. } \\
\text { - } 1 \text { desgarro peritoneal. }\end{array}$ \\
\hline Borzi et al. ${ }^{53}$ & $\begin{array}{l}\text { Selección del } \\
\text { abordaje para } \\
\text { nefrectomia. }\end{array}$ & 179 lap & $\begin{array}{l}\text { Transp. } \\
\text { Retrop. }\end{array}$ & $\begin{array}{l}122 \text { nefrectomías y nefroureterectomías. } 63 \text { nefroureterec- } \\
\text { tomías parciales. TmQ RP 92(25-145) y TP+ureterectomía/ } \\
\text { reconstruc vesical } 153(105-355) \text {. EM RP y TP } 1.5 \text { dias } \\
\text { (1-4), TP complic } 3.5 \text { dias (2-8). Pacientes menos } 5 \text { años, } \\
\text { pielón sup no refluyente, estenosis UPU no funcionante-RP } \\
\text { posterior. Más } 5 \text { años, grandes megauréteres-RP lateral. } \\
\text { Disecciones complejas y pequeños uréteres ectópicos-TP. } \\
\text { Ambas vías son beneficiosas para patologías renales. }\end{array}$ & $\begin{array}{l}6(3.2 \%) \text { conversiones: } \\
\text { - Riñon congelado, perforación duodenal, } \\
\text { mala colocación balón dilatador, } \\
\text { riñon alto en obeso, avulsión vena } \\
\text { renal y sangrado polo inf renal. } \\
\text { - } 4 \text { seromas } \\
\text { - } 1 \text { debito purulento por orificio trocar } \\
\text { - } 3 \text { enfisema }\end{array}$ \\
\hline
\end{tabular}

Tabla 9. Varios

\begin{tabular}{|c|c|c|c|c|c|}
\hline Ref. & Objeto & $\mathbf{n}$ & Abord. & Resultados & Complicaciones \\
\hline $\begin{array}{l}\text { El-Ghoneimi } \\
\text { et al. }{ }^{54}\end{array}$ & $\begin{array}{l}\text { NL en niños de } \\
\text { alto riesgo (Enf. } \\
\text { Renal terminal). }\end{array}$ & $\begin{array}{l}12 \text { lap } \\
12 \text { abt }\end{array}$ & Retrop. & $\begin{array}{l}\text { ASA III todos los casos.Estadío final de enf renal, HTA, } \\
\text { Trombocitopenia +/- S. Nefrótico. Tamaño medio } 8 \mathrm{~cm} \\
\text { Todos los casos fueron bien, no reconversiones. } \\
\text { EM 5,2 días. }\end{array}$ & $\begin{array}{l}\text { - } 3 \text { desgarros peritoneales } \\
\text { - } 1 \text { trombocitopenia.(trans.plaquetas)a } \\
\text { - } 1 \text { hematoma postoperatoria }\end{array}$ \\
\hline $\begin{array}{l}\text { Franc-Guimond } \\
\text { et al. }{ }^{55}\end{array}$ & $\begin{array}{l}\text { Valoración de la } \\
\text { variante de la } \\
\text { técnica de Hasson. }\end{array}$ & 77 lap & Transp. & $\begin{array}{l}\text { Testículos no descendidos (52), varicoleces (11), } \\
\text { nefrectomías (6), nefroureterectomías (1) y otros ( } 7 \text { ). } \\
\text { Edad media 4,8años ( } 1 \mathrm{~mm}-17,75 \text { años). Media seguimiento } \\
15,9 \text { meses. Ventajas en el abordaje incluso en pacientes } \\
\text { difíciles como los obesos. }\end{array}$ & \\
\hline Micali et al. ${ }^{56}$ & $\begin{array}{l}\text { Experiencia con } \\
\text { trócar visul } \\
\text { Visiport. }\end{array}$ & 31 lap & Retrop. & $\begin{array}{l}22 \text { biopsias renales, } 5 \text { varicoceles, } 3 \text { quistes renales, } \\
1 \text { pielolitotomía. Sangrado minimo, Tm } 4 \text { h pielolitotomía } \\
\text { y }<1 \text { hora el resto. EM 1,5 días. Es sencilla y segura } \\
\text { No requiere experiencia. }\end{array}$ & $\begin{array}{l}2 \text { complicaciones menores: } \\
1 \text { - Laceración peritoneal-tto conservador } \\
1 \text { - Arritmia-interrupción procedimiento. }\end{array}$ \\
\hline
\end{tabular}

Dentro de las heminefrectomías/hemiureterectomías laparoscópicas, Valla et al. ${ }^{35}$ describen $3(12 \%)$ reconversiones, 9(37\%) complicaciones intraoperatorias y $5(20 \%)$ complicaciones postoperatorias; Castellan et al. tuvieron 2 conversiones en abordaje retroperitoneal y 2 complicaciones mayores y una menor en el abordaje transperitoneal $^{36}$, y Wallis et al. describieron 4 reconversiones, 5 complicaciones mayores y una complicación mayor ${ }^{38}$.

Desde 1994 a 2007 distintos estudios ${ }^{40-52}$ analizan la efectividad de la laparoscopia en diferentes indicaciones como nefrectomías, heminefrectomías, quistes renales, varicocele, criptorquidia, hidroceles/hernias, biopsias renales, adrenalectomías, reimplante ureteral, pielolitotomías, incluso en uréter retrocavo ${ }^{40}$. En estas series se han descrito 25 recon- versiones ${ }^{40-43,50-53}$ de las que $7(28 \%)$ fueron por sangrado $^{41,43,50-52}$. Los distintos autores consideran este abordaje comparable a la cirugía abierta para el tratamiento de la patología uropediátrica ${ }^{40-42}$.

La Tabla 9 recoge varios artículos que estudian diversos aspectos de la laparoscopia infantil, como el uso del trócar de Hasson y del trócar visul Visiport o cirugías laparoscópicas en niños de alto riesgo ${ }^{52-54}$.

\section{DISCUSIÓN}

La cirugía laparoscópica constituye actualmente una técnica plenamente implantada en la cirugía urológica infantil. Su comienzo y desarrollo ha sido lento debido a la falta de material adecuado y al lento aprendizaje, presentando además diferentes complicaciones durante este periodo, algunas de 
ellas importantes. En nuestra serie ha ocurrido lo mismo, pasando de una vía de abordaje a otra por la presencia de complicaciones, volviéndose a utilizar ambas una vez acumulada la suficiente experiencia y aumentando el número de indicaciones.

No hay acuerdo todavía sobre qué vía de abordaje es mejor. En la revisión de los 45 artículos seleccionados, 18 grupos (38\%) utilizan la vía retroperitoneal, 19 (40\%) la transperitoneal y 10 (21\%) utilizan ambas vías. En nuestra serie hay un predominio claro de la vía transperitoneal (91\%) sobre la retroperitoneal debido principalmente a la aparición de complicaciones en la utilización de ésta última, hecho que se ha ido subsanando con el aumento de experiencia, volviéndose a utilizar ambas según la patología tratada. En principio parece que el urólogo infantil debe sentirse más cómodo en la vía retroperitoneal, probablemente por la similitud con el acceso principal de la cirugía abierta, y además esta vía es segura, presenta buena accesibilidad, riesgo reducido de daño visceral en la introducción de los trócares y facilidad al crear la neumocámara, lo que permite una mínima movilización del riñón con acceso directo al hilio ${ }^{12}$. A pesar de éstas ventajas y su baja morbilidad ${ }^{4}$, este abordaje no es sencillo y requiere un entrenamiento en la técnica por el reducido espacio y por la fragilidad del peritoneo ${ }^{18,45}$. La vía transperitoneal, se considera más apropiada en niños pequeños, ya que ofrece un campo quirúrgico amplio. Cuando se comparan ambas vías, no hay diferencia significativa en cuanto al tiempo quirúrgico, complicaciones o estancia hospitalaria ${ }^{38,7}$. Creemos que el acceso transperitoneal es óptimo para la cirugía renal laparoscópica porque el riesgo de daño visceral, a la hora de crear el neumoperitoneo, es reducido si el abordaje del primer puerto se realiza de forma abierta a través del ombligo, permitiendo además un buen control de los vasos y disminuyendo la probabilidad de daño de otras estructuras por la amplia pneumocámara que se crea, facilitando la ureterectomía completa en caso de ser necesario, sin necesidad de nuevas incisiones, asociándose una menor tasa de reconversiones frente a la retroperitoneoscopia ${ }^{4,8,12}$.

Por regla general, respecto a la cirugía abierta, la laparoscopia es una técnica segura con escaso sangrado y mejora de la estancia hospitalaria, disminuyendo los requerimientos analgésicos y mejorando los resultados estéticos tanto para las heminefrectomías, nefrectomías y nefroureterectomías ${ }^{11-18}$.
Normalmente, en un principio, requiere un mayor tiempo quirúrgico, aunque con el aumento de la experiencia y dominio de la técnica se iguala a las abiertas, pero todavía presenta un mayor coste. En nuestro estudio el tiempo medio quirúrgico varió de 111 minutos a 240 minutos, disminuyendo con el número de casos y el aumento de experiencia.

Las complicaciones que presenta son diversas y disminuyen con el tiempo de experiencia. En la revisión bibliográfica dentro de estos artículos que comparan la cirugía abierta con la cirugía laparoscópica únicamente un grupo no ha tenido complicaciones significativas en el grupo de la laparoscopia ${ }^{17}$; el resto han descrito: conversiones a cirugía abierta, sangrados y/o urinomas ${ }^{12,11,13,14,15}$. Como hemos referido, nosotros hemos tenido un 14\%, considerando el 5,5\% como mayores: urinomas y pseudoaneurisma. La mayoría se presentaron en la primera fase de utilización de la técnica, disminuyendo con el tiempo. No encontramos en la literatura ninguna referencia a la complicación del pseudoaneurisma. Para evitar daños con el electrocauterio actualmente hemos limitado el uso de la pinza gancho, sustituyéndola por la pinza Ligasure $^{\circledR}$ o el bisturí Harmónico desde el comienzo de la intervención, sin nuevas complicaciones asociadas a este instrumental. Recalcamos que la utilización de la pinza Ligasure $^{\circledR}$ nos ha dado unos resultados excelentes, con un sagrado mínimo y un buen control del pedículo. La energía de coagulación y después el corte con la cuchilla sucede entre las dos ramas de la pinza sin que difunda más allá de las mismas. No obstante el grosor de la punta roma de la pinza dificulta disecciones complicadas. De forma semejante a nosotros, la complicación más frecuente encontrada en la bibliografía ha sido el urinoma tras heminefrectomía o nefrectomía ${ }^{11-13,19,34,35,37,40}$ seguido de otras menores como los desgarros peritoneales $^{13,26,35,40,41,44,54,56}$ o el daño visceral ${ }^{15,35,40,41}$. La mayoría de los urinomas se han resuelto de forma conservadora y sólo en dos casos se requirió drenaje percutáneo ${ }^{12,35}$. Las causas más frecuentes de reconversión a cirugía abierta han sido el control $\operatorname{vascular}^{41,44,53}$, el sangrado ${ }^{17,19,29,44,53}$ o la dificultad técnica $29,37,51$.

Nuestro programa de laparoscopia ha estado orientado, principalmente, a la patología benigna renal, efectuando nefrectomías, nefroureterectomías y heminefrectomías, comenzando en los últimos 2 años con patología tumoral como los nefromas 
mesoblásticos y neuroblastomas. El papel diagnóstico de la laparoscopia está bien establecido aunque siguen en discusión ciertas indicaciones terapéuti$\operatorname{cas}^{9,42}$. Los buenos resultados estéticos y su rápida recuperación sugieren que los niños y adolescentes pueden beneficiarse de ella. Sin embargo se cuestiona la utilidad real de esta técnica en menores de 5 años y lactantes, en especial los menores de un año ${ }^{40}$. Los varicoceles, criptorquidias, estados intersexuales y la cirugía renal, incluyendo procedimientos como la nefrectomía, heminefrectomía, nefropexia, quistectomía, pieloplastia y adrenalectomía constituyen indicaciones actuales de la cirugía laparoscópica ${ }^{9,43,56}$. Otras cirugías reconstructivas y las exéresis tumorales se están introduciendo como nuevas indicaciones ${ }^{40,41,43,51}$. Duarte et al. confirman que la nefrectomía laparoscópica tras quimioterapia neoadyuvante es segura en casos seleccionados. La laparoscopia infantil es aceptada en la resolución de patologías benignas pero su uso aún está poco contrastado en la cirugía oncológica ${ }^{21}$. Además la cirugía robótica está en proceso inicial de desarrollo para empezar a ser aplicada en la cirugía infantil.

Basándonos en lo publicado en las series revisadas, la cirugía laparoscópica es el estándar de tratamiento en la mayoría de los casos de patología benigna infantil. En manos experimentadas es segura y efectiva en niños de alto riesgo y en menores de un año ${ }^{32,55,56}$. La diversificación de la laparoscopia pasa por generalizar programas de aprendizaje para urólogos infantiles en la fase de formación laparoscópica ${ }^{10,12,18,23,33,31,43}$. Es importante evitar problemas derivados de un defecto en la formación que puede conllevar una alta incidencia de complicaciones, lo que originaría un descredito de la técnica. En nuestra opinión es necesario un entrenamiento teórico e ir adquiriendo experiencia en dispositivos endourológicos o sobre animales de experimentación, antes de proceder sobre pacientes. El equipo quirúrgico ha de maximizar el valor educacional de cada una de las operaciones para los nuevos cirujanos laparoscopistas. La experiencia del cirujano mejora el tiempo quirúrgico, el sangrado y disminuye la tasa de morbilidad $^{14,23,38}$.

En conclusión los tiempos quirúrgicos, la estancia y la tasa de complicaciones se han igualado, incluso mejorando a las de la cirugía abierta. Previo a su aplicación en el paciente, el cirujano ha de adquirir las destrezas necesarias en programas específicos de aprendizaje que minimicen las complicaciones. En manos experimentadas, la laparoscopia infantil, se muestra como una técnica segura y fiable en el tratamiento de las patologías benignas urológicas, con resultados comparables a la cirugía convencional. Actualmente otros campos como la cirugía reconstructiva y la oncológica se están aplicando, describiéndose buenos resultados, además la cirugía robótica ya se está aplicando en diferentes patologías, pero aún precisa de grandes medios técnicos y tiene un coste elevado.

Abreviaturas: EM: Estancia media. TmQ: Tiempo medio quirúrgico. L: Cirugía Laparoscópica. A: Cirugía abierta. Transp: Abordaje transperitoneal. Retrop: Abordaje retroperitoneal.

\section{REFERENCIAS}

1. Clayman RV, Kavoussi LR, Soper NJ, Dierks SM, Meretyk S, Darcy MD, et al. Laparoscopic nephectomy : Initial case report. J Urol. 1991;146(2):278-282.

2. Jordan GH, Winslow BH. Laparoscopic upper pole partial nephectomy with ureterectomy. J Urol. 1993;150(3):940-943.

3. Luque Mialdea R, Martin-Crespo R. Laparoscopy in pediatric urology. Arch Esp Urol. 2002;55(6):737-47.

4. Valla JS, Colomb F, Son S, Michelini E, Steyaert H, Guilloneau B. Mini-invasive surgery of the retroperitoneal space in children. Ann Urol (Paris). 1999;33(5):328-332.

5. Wallis MC, Khoury AE, Lorenzo AJ, Pippi-Salle JL, Bägli DJ, Farhat WA. Outcome analysis of retroperitoneal laparoscopic heminephectomy in children. J Urol. 2006;175(6):2277-2282.

6. http://www.cebm.net/. Oxford Centre for Evidence-based Medicine levels of evidence.

7. Kim C, McKay K, Docimo SG. Laparoscopic Nephrectomy in Children: Systematic Review of transperitoneal and Retroperitoneal Approaches. Urology. 2009;73(2):280-284.

8. Borzi P, Yeung CK. Selective approach for transperitoneal and extraperitoneal endoscopio nephectomy in children. J Urol. 2004; $171(2$ Pt 1):814-816.

9. Peters CA. Laparoscopy in pediatric urology. Curr Opin Urol. 2004;14(2):67-73.

10. Robinson BC, Snow BW, Cartwright PC, De Vries CR, Hamilton $\mathrm{BD}$, Anderson JB, et al. Comparison of laparoscopic versus open partial nephrectomy in pediatric series. J Urol. 2003;169(2):638640.

11. Lee RS, Retik AB, Borer JG, Diamond DA, Peters CA. Pediatric retroperitoneal laparoscopic partial nephrectomy: comparison with an age matched cohort of open surgery. J Urol. 2005; 174(2): 708-712.

12. Piaggio L, Franc-Guimond J, Figueroa E, Barthold JS, González R Comparison of laparoscopic and open partial nephrectomy for duplication anomalies in children. J Urol. 2006;175 (6):2269-2273.

13. El-Ghoneimi A, Farhat W, Bolduc S, Bagli D, McLorie G, Khoury A. Retroperitoneal laparoscopic vs open partial nephroureterectomy in children. BJU Int. 2003;91(6):532-535.

14. Chertin B, Ben-Chaim J, Landau EH, Koulikov D, Nadu A, Reissman P,. Pediatric transperitoneal laparoscopic partial nephrectomy: comparison with an age-matched group undergoing open surgery. Pediatr Surg Int. 2007;23(12):1233-1236.

15. Hamilton BB, Gatti MG, Cartwrright PC, Snow BW. Comparison of laparoscopic versus open nephrectomy in the pediatric population. J Urol. 2000;163(3):937-939. 
16. Ku JH, Yeo WG, Choi H, Kim HH. Comparison of retroperitoneal laparoscopic and open nephrectomy for benign renal diseases in children. Urology. 2004;63(3):566-70.

17. Sekaran P, MacKinlay GA and Lam J. Comparative evaluation of laparoscopic versus open nephrectomy in children. Scott Med J. 2006;51(4):15-17.

18. Valla JS, Guilloneau B, Montupet P, Geiss S, Steyaert H, el Ghoneimi A, et al. Retroperitoneal laparoscopic nephrectomy in children: preliminary report of 18 cases. Eur Urol. 1996;30(4): 490-349.

19. Kobashi KC, Chamberlin DA, Rajpoot D, Shanberg AM. Retroperitoneal laparoscopic nephrectomy in children. J Urol. 1998; 160(3 Pt2): 1142-1144.

20. Davies BW, Najmaldin AS. Transperitoneal laparoscopic nephrectomy in children. J Endourol. 1998;12(5):437-440.

21. Yao D, Poppas DA clinical series of laparoscopic nephrectomy, nefphroureterectomy and heminephroureterectomy in the pediatric population. J Urol. 2000;163(5):1531-1515.

22. Dronov AF, Poddubny凶 IV, Smirnov AN, Kovarski冈 SL, Korznikova IN, Al-Mashat NA, et al. Videolaparocopic nephectomy and nephouretherectomy in children. Khirurgiia. 2004;(2):43-47.

23. Ku JH, Yeo WG, Kim HH, Choi H.. Laparoscopic nephrectomy for renal diseases in children: is there a learning curve?. J Pediatr Surg. 2005;40(7): 1173-1176.

24. Mahomed AA, Hoare C, Welsh F, Driver CP. A two-center experience with the exclusive use of laparoscopic transperitoneal nephrectomy for benign renal disease in children. Surg Endosc. 2007;21(9): 1532-1526.

25. Jeong BC, Lim DJ, Lee SC, Choi H, Kim HH. Laparoscopic nephrectomy for a single-system ectopic ureter draining a small, dysplastic and poorly functioning kidney in children. Int $\mathrm{J}$ Urol. 2007; 14(2): 104-107.

26. Gundeti MS, Taghizaedh A, Mushtaq I. Bilateral synchronous posterior prone retroperitoneoscopic nephrectomy with simultaneous peritoneal dialysis: a new management for end-stage renal disease in children. BJU. 2007;99(4):904-906.

27. Jesch NK, Metzelder ML, Kuebler JF, Ure BM. Laparoscopic transperitoneal nephrectomy is feasible in the in the first year of life and is not affected by kidney size. J Urol. 2006;176(3):1177-1179.

28. Duarte RJ, Dénes FT, Lilian MC Odone-Filho V, Srougi M. Further experience with laparoscopic nephrectomy for Wilms tumour after chemotherapy. BJU Int. 2006;98(1):155-159.

29. Lam JP, MacKinlay GA, Munro FD, Aldridge LM. Endoscopic nephrectomy in children. Is retro the way forward?. J Laparoendosc Adv Surg Tech A. 2006;16(1):59-62.

30. Steven LC, Li AGK, Driver CP, Mahomed AA. Laparoscopic nephrectomy for unilateral multicystic dysplastic kidney in children. Surg endosc. 2005;19(8):1135-1138.

31. Janetschek G, Seibold J, Christian R, Bartsch G. Laparoscopic heminephroureterectomy in pediatric patients. J Urol. 1997;158 (5):1928-1930.

32. Borer JG, Cisek LJ, Atala A, Diamond DA, Retik AB, Peters CA. Pediatric retroperitoneoscopic nephrectomy using $2 \mathrm{~mm}$ instrumentation. J Urol. 1999;162(5):1731-1732.

33. York GB, Robertson FM, Cofer BR, Bomalaski MD, Lynch SC. Laparoscopic nephectomy in children. Surg Endosc. 2000;14(5): 469-472.

34. Horowitz M, Shah SM, Ferzli G, Syad PI, Glassberg KI. Laparoscopic partial upper pole nephrectomy in infants and children. BJU Int. 2001;87(6):514-516.

35. Valla JS, Breaud J, Carfagna L, Tursini S, Steyaert H. Treatment of ureterocele on duplex ureter: upper pole nephrectomy by retroperitoneoscopy in children based on a series of 24 cases. Eur Urol. 2003;43(4):426-429.

36. Castellan M, Gosalbez R, Carmack AJ, Prieto JC, Perez-Brayfield M, Labbie A. Transperitoneal and retroperitoneal laparoscopic heminephrectomy what approach for which patient?. J Urol. 2006;176(6 Pt 1):2636-2639.
37. Wallis MC, Khoury AE, Armando JL et al. Outcome análysis of retroperitoneal laparoscopic heminephrectomy in children. J Urol 2006; 175:2277-82.

38. Yucel S, Brown B, Bush NC et al. What to anticipate with experience in pediatric laparoscopic ablative renal surgery. J Urol 2008; 179:697-702.

39. Dénes FT, Danilovic A and Srougi M. Outcome of laparoscopic upper-pole nephrectomy in children with duplex systems. J Endourol 2007;21:162-9.

40. Valla JS, Colomb F, Son S et al. Mini-invasive surgery of the retroperitoneal space in children. Ann Urol (Paris) 1999;33(5): 328-32.

41. El-Ghoneimi A, Valla JS, Steyaert H, Aigrain Y. Laparoscopic renal surgery via a retroperitoneal approach in children. J Urol. 1998;160(3 Pt2):1138-1141.

42. Luque Mialdea R, Martín-Crespo R. Laparoscopy in pediatric urology. Arch Esp Urol. 2002;55(6):737-747.

43. Seibold J, Janetschek G, Bartsch G. Laparoscopic surgery in pediatric urology. Eur Urol. 1996;30(3):394-9.

44. Shanberg AM, Sanderson K, Rajpoot D, Duel B. Laparoscopic retroperitoneal renal and adrenal surgery in children. BJU Int. 2001;87(6):521-524.

45. Ehrlich RM, Gershman A, Fuchs G. Laparoscopic renal surgery in children. J Urol. 1994;151(3):735-739.

46. Tadini B, Repetto L, Guarino N et al. Retroperitoneoscopic renal surgery in children: our experience. J Laparoendosc Adv Surg Tech A. 2006;16(3):305-307.

47. Fahlenkamp D, Winfield HN, Schönberger B et al. Role of laparoscopic surgery in pediatric urology. Eur Urol 1997;32(1):75-84.

48. Urbanowicz W, Wieczorek M and Sulislawski J. Retroperitoneoscopic nephrectomy in the prone position in children (point of technique). Eur Urol. 2002;42(5):516-519.

49. Gundeti MS, Patel Y, Duffy PG, Cuckow PM, Wilcox DT, Mushtaq I. An inicial experience of 100 paediatric laparoscopic nephrectomies with transperitoneal or posterior prone retroperitoneoscopic approach. Pediatr Surg Int. 2007;23(8):795-799.

50. Mulholland TL, Bradley PK, Wong C. Laparoscopic renal surgery in infants $10 \mathrm{~kg}$ or less. J Endourol 2005;19(3):397-400.

51. Farhat W, Khoury A, Bagli D, McLorie G, El-Ghoneimi A. Mentored retroperitoneal laparoscopic renal surgery in children: a safe approach to learning. BJU Int 2003;92(6):617-620.

52. Capolicchio J, Jednak R, Anidjar M, Pippi-Salle JL. A modified access technique for retroperitoneoscopic renal surgery in children. J Urol. 2003;170(1):204-206.

53. Borzi PA, Yeung CK. Selective approach for transperitoneal and extraperitoneal endoscopic nephrectomy in children. J Urol. 2004;171(2 Pt 1):814-816.

54. El-Ghoneimi A, Sauty L, Maintenant J, Macher MA, Lottmann H, Aigrain Y. Laparoscopic retroperitoneal nephrectomy in hig risk children. J Urol. 2000;164(3 Pt 2):1076-1079.

55. Franc-Guimond J, Kryger J, González R. Experience with the Bailez technique for laparoscopic access in children. J Urol. 2003;170(3):936-938.

56. Micali S, Caione P, Virgili G, Capozza N, Scarfini M, Micali F. Retroperitoneal laparoscopic access in children using a direct vision technique. J Urol. 2001;165(4):1229-1232.

Correspondencia: Dr. Alberto Pérez-Lanzac de Lorca

Servicio de Urología

Hospital Universitario Virgen Macarena

Avda. Dr. Fedriani, 3 - 41071 Sevilla.

Tel.: 955008265

E-mail autor: alblorca@yahoo.es

Información artículo: Original - Pediatría

Trabajo recibido: marzo 2009

Trabajo aceptado: abril 2009 